$\operatorname{IPPP} / 02 / 24$

$\mathrm{DCPT} / 02 / 48$

\title{
Multiparticle Production in QCD Jets
}

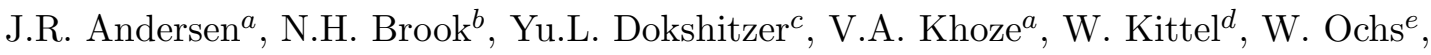 \\ W.J. Stirling ${ }^{a}$ and G. Zanderighi ${ }^{a}$ \\ ${ }^{a}$ IPPP, Department of Physics, University of Durham, Durham DH1 3LE, UK \\ ${ }^{b}$ H.H. Wills Physics Laboratory, University of Bristol, Bristol BS8 1TL, UK \\ ${ }^{c}$ LPTHE, Université Pierre et Marie Curie (Paris VI), 75252 Paris, France \\ ${ }^{d}$ HEFIN, University of Nijmegen/NIKHEF, 6515 ED Nijmegen, The Netherlands \\ e Max Planck Institut für Physik, D-80805 Munich, Germany
}

\begin{abstract}
We briefly summarise the main results presented at the IPPP Workshop on Multiparticle Production in QCD Jets, held in Durham in December 2001.
\end{abstract}




\section{Introduction}

During the last few years, particle physics experiments have collected an impressive amount of new information on multiparticle production in hadronic jets, which allows many important tests of both perturbative and nonperturbative aspects of QCD to be performed. It is therefore timely to survey the current status of such studies and to consider future directions for work in this field.

The main aim of the IPPP Workshop on "Multiparticle Production in QCD Jets" was to survey recent theoretical results and the experimental data from the LEP era, HERA and the Tevatron. Particular attention was paid to the prospects for QCD studies at the LHC and at a future linear $e^{+} e^{-}$collider.

\section{Jet Production and Fragmentation}

The D0 [1] and CDF [2] collaborations presented results on jet fragmentation at the Tevatron, in particular on the momenta spectra of charged particle distributions and the subjet multiplicities.

The CDF collaboration have investigated [3] the momenta spectra of charged particles in central dijet (or $\gamma$ - jet) events with a dijet mass in the range $80<M_{j j}<630 \mathrm{GeV}$. To limit any biases being introduced, due to the fact that there is underlying event debris in the hadron collider environment, the analysis is confined to small opening angles around the jet axis. The scaled momentum distribution of the charged tracks, $\xi=\log \left(E_{\text {jet }} / p\right)$, associated with a jet was measured and compared to the Modified Leading Logarithmic Approximation (MLLA) parton predictions [⿶]. The parton predictions are related to the hadron distributions, according to the Local Parton Hadron Duality (LPHD) picture [5], by a simple normalisation factor, $K$. In the MLLA theory the dependence of the $\xi$ distribution on the jet energy and cone angle, $\theta$, is given by the variable $Y=\log \left(E_{\text {jet }} \sin \theta / Q_{\text {eff }}\right)$, where $Q_{\text {eff }}$ is a cut-off scale for the gluon emissions. The CDF fits to the data confirm the scaling of the momenta spectra with $E_{\text {jet }} \sin \theta$ with a $Q_{\text {eff }}=240 \pm 40 \mathrm{MeV}$.

The CDF collaboration have also studied the evolution of the charged particle multiplicity with $M_{j j}$. Using the value of $Q_{\text {eff }}$ obtained above from the momenta spectra and the fraction of gluon jets in the dijet sample extracted from the HERWIG Monte Carlo generator [6], it is possible to extract the value of $K$ and the ratio of parton multiplicities in gluon and quark jets, $r$. The fit yielded a value of $K=0.57 \pm 0.06 \pm 0.09$ and $r=1.7 \pm 0.3$. The choice of parton density functions (which affects the fraction of gluon jets) and $Q_{\text {eff }}$ have negligible effect on the value of $r$ but are a large contribution to the systematic errors on $K$. This value of $K$ only reflects the ratio of the number of charged particles to the number of partons and is consistent with the LPHD hypothesis of a one-to-one correspondence between final partons and all observed hadrons. The value of $r$ is also consistent with that expected from MLLA.

The D0 collaboration have also studied the internal jet structure through resolving subjets [7]. The motivation was again to study the difference between quark and gluon jets. To achieve this it was necessary to select enriched quark and gluon jet samples. This was realised by probing the internal structure of jets at the same $E_{T}$ but from $\mathrm{p} \overline{\mathrm{p}}$ collisions at different centre of mass energies $(\sqrt{s}=630$ and $1800 \mathrm{GeV})$. For jets with $55<p_{T}$ (jet) $<100 \mathrm{GeV}$ a gluon enriched sample is found at the higher $\sqrt{s}$ compared to a quark fraction that is enhanced at the lower

\footnotetext{
*Author: N. Brook
} 
$\sqrt{s}$. The relative fraction of quark/gluon events is estimated using the HERWIG Monte Carlo generator.

The $k_{T}$ jet algorithm [8] was used in this analysis; in addition to the $p_{T}$ criteria listed above, the jets were required to be centrally produced, i.e. have a pseudorapidity $|\eta|<0.5$. The subjet multiplicity was calculated by using the "pre-clusters" associated with a particular jet and reapplying the jet algorithm and using a jet resolution parameter equivalent to the minimum subjet $p_{T}$ being $\sim 3 \%$ of the total jet $p_{T}$. The average subjet multiplicities were measured to be $M_{1800}=2.74 \pm 0.01$ and $M_{630}=2.54 \pm 0.03$ at $\sqrt{s}=1800$ and $630 \mathrm{GeV}$ respectively. Applying corrections for detector effects and the fraction of gluon events at each $\sqrt{s}$, the mean subjet multiplicity of a quark jet, $M_{q}=1.69 \pm 0.04$, and a gluon jet, $M_{g}=2.21 \pm 0.03$, can be derived. Furthermore from the fact that $M-1$ corresponds to the average number of subjet emissions a value of $r$ can be extracted again. D0 measured $r=1.84 \pm 0.15 \pm_{0.18}^{0.22}$ with the major systematic error being the determination of the gluon jet fraction. This complementary measurement of $r$ is consistent with the value presented by CDF.

The motivation for limiting the cone size in the CDF analysis of the charged particle distibution was the need to understand the so-called underlying event. The underlying event is defined as everything not associated with the hard two-to-two subprocess. Experimentally the underlying event is measured using minimum bias data - soft inelastic collisions in which both the incoming hadrons break up. It has been shown 9 that the underlying event in hard scattering has considerably more activity than the soft collisions for the same available energy. The model currently implemented in the HERWIG Monte Carlo event generator is based on the minimum bias generator of UA5 and fails to reproduce this additional activity.

Borozan et al. [10] have been developing a model for soft interactions which is founded on a firm theoretical basis. The model is based on an eikonal approach. It takes into account contributions from hard multiparton interactions and a soft component, representing the opaqueness of the hadron as a function of the available energy and impact parameter. This was shown to successfully describe the CDF data at $\sqrt{s}=1800 \mathrm{GeV}$.

At Tevatron energies it is known that the fixed order QCD calculations for heavy quark production underestimate the cross section for $b$-production when compared with the data. Another approach [11] to heavy quark production is that of parton showers (PS). Although only approximate, it has the advantages of resumming large logarithms, being process generic and easy to match to hadronisation. The PS approach convolutes the two-to-two hard scatter with initial state and final state (QCD) radiation to represent the two-to-many final parton configuration. In the PS approach, 3 main contributions to heavy quark production can be isolated:

1. pair creation $(g g \rightarrow Q \bar{Q}$ and $q \bar{q} \rightarrow Q \bar{Q}$ plus additional showering)

2. flavour excitation based on the $c$ and $b$ content of the parton density functions $(Q q \rightarrow Q q$ and $Q g \rightarrow Q g$

3. gluon splitting (e.g. $g g \rightarrow g g$ with $g \rightarrow Q \bar{Q}$.)

By including the contributions to $b$-quark production from the PS it is possible to describe the $b$-quark cross sections in the central rapidity regions as a function of the minimum transverse momentum of the $b$-quark at $\mathrm{CDF}[12]$. 
The hadronisation of heavy quarks should not be studied in isolation from the production environment. There are "beam" remnant issues that affect the $b$ - quark distributions due to "drag" effects. In the string model there are 3 hadronisation mechanisms:

1. normal string fragmentation leading to a continuum of phase-space states,

2. cluster decay, where a low mass string leads to an exclusive two-body state, and

3. cluster collapse, where a very low mass string becomes a single hadron.

These production mechanisms can lead to a production asymmetry between hadrons containing heavy quarks and antiquarks. These production asymmetries have been observed in fixedtarget experiments studying charm production [13]. The modelling of the low-mass clusters is particularly sensitive to these asymmetries and the low-mass behaviour of the models has been refined [14] in order to reproduce the effect observed by experiment. The phenomenological parameters introduced into the framework leave a large leeway in the predictive ability for these asymmetries for $b$-quarks at higher centre-of-mass collisions. It is important that the study of heavy quark production is made in conjunction with the development of the multiple interaction models.

By studying high energy cosmic rays it is hoped to gain insight into their source and acceleration mechanism. Particular features that are under study are the 'knee' in the particle flux spectrum that occurs at $\sim 10^{3} \mathrm{TeV}$ and the precise form of the spectrum at energies $>10^{7} \mathrm{TeV}$. To study cosmic rays at and above these energies it is necessary to understand the properties of the particle showers that are produced as the cosmic ray enters the atmosphere. The mass and energy of the primary particles are deduced from the properties of these air showers. The form of the air showers are dependent on the physics of the hadronic interactions, electromagnetic interactions, particle production, decays and transport mechanisms. The reconstruction of the primary energy and mass is particularly sensitive to the height of the shower maximum, the lateral particle distribution and particle content of the air showers at ground level. The ability to reconstruct the mass is currently limited by the modelling of hadronic and nuclear interactions [15]. The hadronic models being developed are based on Gribov-Regge theory of multi-Pomeron exchange for the dominant soft interactions. Particle production is modelled including string fragmentation. They have to take into account extrapolation to higher energies, diffraction, hard processes and nuclear interactions. The models are beginning to describe the cosmic ray data rather well. The various models available converge: for example the differences in the cross section, $\sigma_{\mathrm{p}-\text { air }}$, are within $8 \%$ and the shower maximum within $6 \mathrm{~g} / \mathrm{cm}^{2}$. Unfortunately there are still areas where the uncertainties are greater than $10 \%$ where it is hoped that future accelerator-based HEP experiments, such as those based at heavy ion facilities, could help in increasing the understanding of the processes involved. The Auger experiment, under construction in Argentina, will soon allow investigation of the hadronic interaction models at energies as high as $10^{8} \mathrm{TeV}$. 


\section{Perturbative and Non-perturbative Aspects of Multiparticle Production!}

The production of multiparticle final states in hard collisions according to the standard description involves three phases: first there is a hard primary interaction at small distance scales with few outgoing partons, leptons or gauge bosons, subsequently the produced partons evolve into parton jets according to a perturbative description, and finally, there is a non-perturbative transition into the observable hadrons at large distances. All three phases of particle production have been addressed at the workshop. The aim is the improvement of the theoretical description, calculations of new observables and the experimental tests of predictions.

\subsection{Improved treatment of primary hard processes}

An improved accuracy is required for a better test of the theory and also for a better understanding of QCD processes in the search for new physics at future colliders. The parton shower calculations as applied in event generators are accurate only in the phase space regions near the soft and collinear singularities but typically fail at large angles where full matrix element results are needed.

For the case of $e^{+} e^{-}$annihilation an algorithm is presented by Lönnblad [16] in which the Colour-Dipole Cascade Model, as implemented in the ARIADne program, is corrected to match the fixed order tree-level matrix elements for $e^{+} e^{-} \rightarrow n$ jets. The result is a full parton-level generator for $e^{+} e^{-}$annihilation where the generated states are correct at tree-level to fixed order in $\alpha_{S}$ and to all orders with MLLA accuracy. In addition, virtual corrections are taken into account to all orders with MLLA accuracy. Results have been presented and discussed, where matrix elements are used up to second order in $\alpha_{S}$. The algorithm is applicable also for higher orders and it should be possible to modify it to work even for hadronic collisions.

Similar problems are met in the computation of hadronic electroweak gauge boson pair production $(\gamma \gamma, Z Z, W W, Z \gamma, W \gamma, W Z)$ as discussed by Burby [17]. These processes are of interest, in particular in the search for the Higgs boson and physics beyond the Standard Model. A complete framework for analyses is provided by parton shower based event generators. It is demonstrated how the description of the high $p_{T}$ emissions in gauge boson pair production is improved by merging the QCD matrix elements with the parton shower algorithm of PYTHIA. A method of overpopulating the shower phase space and then rejecting events down to the matrix element rate is employed by calculation of a correction factor which takes into account the possible parton shower histories. In addition to gluons, real photons are permitted to shower and hence must also be included in the matrix element corrections.

Another problem which occurs in the calculation of radiative QCD corrections to pair production of $W$ and $Z$ bosons, as well as to top quarks, is the finite width of these particles. This has been discussed by Chapovsky [18]. The structure of higher-order radiative corrections for processes with unstable particles is analysed. The mass $M$ and width $\Gamma$ of the unstable particles yield a hierarchy of scales. By subsequently integrating out the modes induced by these scales, a hierarchy of effective field theories is obtained. In the effective field theory framework the separation of physically different effects is achieved naturally. In particular, a separation of factorisable and non-factorisable corrections to all orders in perturbation theory is obtained. The non-factorisable corrections can be classified in two ways. First of all, they can be of the

\footnotetext{
${ }^{\dagger}$ Author: W. Ochs
} 
production-decay and decay-decay types. Second, they can be due to interaction with the production/decay dipoles and propagation corrections. It is known that one-loop non-factorisable corrections to invariant mass distributions are suppressed by powers of $M / E$ at high energies. The mechanism of this suppression is studied and estimates of higher-order non-factorisable corrections at high energy obtained. These results are applied to the above pair production processes.

\subsection{Semisoft processes: experimental tests of perturbative predictions}

Various details of the multiparticle final state concerning particle multiplicities and their inclusive differential distributions have been studied in the recent years and analytical results derived. This involves particles with energies around $1 \mathrm{GeV}$ or smaller. A simple model relates the partonic distributions obtained after an evolution of the parton cascade towards small scales $\left(Q_{0} \sim \Lambda\right)$ directly to the corresponding hadronic distributions. Such a correspondence could hold in a dual sense and was originally proposed for single inclusive spectra (LPHD [5]). Detailed experimental data became available from LEP (for a review see 19]) and more recently from HERA. The status of the model was reviewed by Ochs (see also [20]).

It has been concluded that the agreement between the perturbative predictions and experiment had been generally successful, depending on the accuracy of the calculation. Global quantities like mean multiplicities and correlation moments can be described at the quantitative level. The characteristic features of single inclusive particle distributions ('hump backed plateau') are well established for all types of particles and the approach to asymptotic scaling (' $\zeta$-scaling') follows the DLA (Double Logarithmic Approximation) and MLLA predictions. However the behaviour in the very soft region $\left(k_{T} \sim Q_{0}\right)$ depends on mass effects and additional model assumptions are needed to obtaim a good description. Nevertheless, the early onset of asymptotic behaviour of this ultrasoft region is generic and its verification supports the underlying soft gluon coherence and 'angular ordering'. Angular correlations agree with the various asymptotic (DLA) predictions at least qualitatively and with numerical results at the almost quantitative level. Calculations concerning the transition $y_{c u t} \rightarrow 0$ from jet to hadron and the transition to quasi-exclusive processes involving large rapidity gaps have been surprisingly successful. A problem has recently been met with multi-correlations of soft $\left(k_{T} \sim Q_{0}\right)$ particles (see below).

In most applications of this type the relative momenta can become small and therefore the coupling $\alpha_{s}\left(k_{T}\right)$ can become large of order 1 . The extension of the calculations into this kinematic region is not justified a priori, but the perturbative expansion is converging rapidly and one can consider the overall successful description at least as an effective parametrisation of the soft part. Remarkably, the coupling $\alpha_{s}$ obtained at small scales is roughly consistent with the experimental results for the integral $1 / \mu \int_{0}^{\mu} \alpha_{s}(k) d k$ obtained from fits to shape variables using the perturbative approach with power corrections added (see Section 5 below).

Global moments of multiplicity distributions of quark and gluon jets, in particular their mean values $N_{F, G}$, have been computed by solving the so-called MLLA evolution equations, as discussed by Dremin [21] (for a recent review [22]). At higher energies the solutions can be written as $N_{F, G} \propto \exp \left(\int^{y} \gamma_{F, G}\left(y^{\prime}\right) d y^{\prime}\right)$ with the anomalous dimensions $\gamma_{F, G}$ calculated in powers of $\gamma_{0} \propto \sqrt{\alpha_{S}}$, where the LO and NLO terms for $N_{F}$ and $N_{G}$ are the same. The ratio $r=N_{G} / N_{F}$ has been calculated to order $\gamma_{0}^{3}$, which corresponds to the inclusion of the 4 NLO expansion of $\gamma_{G, F}$. Asymptotically $r \rightarrow C_{A} / C_{F}=9 / 4$, and at LEP energies with increasing 
accuracy the calculations approach the data and the exact numerical solutions of the evolution equations finally give good agreement. It is advantageous to study the quantities $r^{(1)}=N_{G}^{\prime} / N_{F}^{\prime}$ and $r^{(2)}=N_{G}^{\prime \prime} / N_{F}^{\prime \prime}$ as they have small higher order corrections. QCD predicts

$$
r<r^{(1)}<r^{(2)}<C_{A} / C_{F}=2.25 .
$$

The experimental determinations of the ratio $C_{A} / C_{F}$ from jet multiplicities and from scaling violations of particle spectra by DELPHI have been explained by Siebel [23]. The energy dependence of the jet multiplicities $N_{F}$ and $N_{G}$ is determined from quark and gluon jets isolated in $e^{+} e^{-} \rightarrow 3$ jets using certain jet algorithms. The above ratio $r^{(1)}$ has been determined this way and the results are less sensitive to non-perturbative effects than the absolute values of the multiplicities. The relation (11) has been confirmed by this procedure.

In an alternative approach, the total multiplicity of 3 -jet events as a function of jet angles is compared to the theoretical description which does not assign particles to individual jets but rather takes into account the coherent emission from the $q \bar{q} g$ 'antenna'. The results from both methods are consistent with the QCD expectation $C_{A} / C_{F}=9 / 4$.

The 'classical' MLLA prediction of the hump-backed plateau, namely the Gaussian type distribution of particles in the variable $\xi=\log \left(1 / x_{p}\right)$ (with $x_{p}=p / p_{\max }$ determined in the current region of the Breit frame) has been scrutinised by Brook using data from LEP and HERA [24]. The moments have been extracted from fits of a distorted Gaussian to data around the maximum and compared to several theoretical predictions which differ in their treatment of soft particles (large $\xi$ ). The data are bracketed by the MLLA predictions with and without inclusion of a mass effect (taking $Q_{0}$ as the particle mass). A good description can be obtained by introducing an additional effective mass parameter. At high energies all descriptions converge and agree with the data. It should be recalled here, that the MLLA description is not very good for soft particles because of slow convergence of the $\sqrt{\alpha_{S}}$ expansion, and better results can be obtained by using the first two terms of the perturbative expansion.

The recent results from LEP and HERA on multiparticle angular correlations have been discussed by Chekanov. In general, two-particle densities and angular correlations in DIS jets agree with the DLA calculations [25]. The normalised factorial moments, measured in rings around the jet direction, show discrepancies with the perturbative QCD predictions for all three experiments, L3 [26], DELPHI [27] and ZEUS [28]. From parton-level Monte-Carlo studies, it was concluded that these discrepancies are related to the approximations inherent in the analytical DLA results (especially neglect of energy-conservation constraints). A more serious discrepancy with the theoretical predictions has been found recently for correlations in restricted $p_{t}$ and $p$ regions by ZEUS 28]. This is the first example where perturbative QCD, in conjunction with the LPHD hypothesis, fails on a qualitative level. As in case of the single particle spectra, the problem is with particles near the non-perturbative boundary $p_{t} \sim Q_{0}$ where mass effects become important. It is suggested elsewhere that the predictions should work for mini-jets with low virtuality around $Q_{c} \sim 1 \mathrm{GeV}$.

\subsection{Non-perturbative models}

At HERA and the TEVATRON sizeable fractions (10\% and $1 \%$ respectively) of events with two high $E_{T}$ jets have a large rapidity gap. A possible explanation of such events is the presence of a hard colour singlet exchange. Metzger [29] reported on an analysis of $e^{+} e^{-} \rightarrow q \bar{q} g$ events 
from hadronic $Z$ decays obtained by the L3 collaboration to search for additional evidence for the presence of this mechanism.

In the JETSET parton shower model of this process, hadrons are produced from two strings connecting the gluon with the quark and the antiquark. Models with colour singlet exchange are constructed in which a string is built up between the $q \bar{q}$ pair, whereas the gluon is disconnected from the quarks and in one example hadronises like a boosted $q \bar{q}$ pair. Particle flows between the three jets are found to be sensitive to the presence of the colour singlet mechanism. The result of this study is that the data can accommodate no more than about $8 \%$ colour-singlet exchange (at 95\% CL). Furthermore, the data are found to be incompatible with the recently proposed model by Rathsman assuming a colour connection based upon a generalised Lund area scheme. Similar methods are applied to four-jet events in $e^{+} e^{-} \rightarrow W^{+} W^{-}$to place limits on various colour reconnection models.

The possibility of another non-perturbative phenomenon in jet evolution, squeezed states, was considered by Kuvshinov [30]. This phenomenon is known in quantum optics and can be obtained from coherent states. The time evolution of a gluon coherent state is studied using a QCD Hamiltonian approach. It is shown that after a small period of time, squeezed states appear due to the self-interaction of the gluon quantum. The gluon correlation function has singularities at very small angles. Furthermore, there are connections between squeezing and a chaotic behaviour of gluons in the jet.

\section{Correlations and Fluctuations}

Analytical QCD calculations at the DLA and/or MLLA level, combined with the LPHD hypothesis [5], have proven particularly successful in explaining single-particle distributions and the energy evolution of their integrals, i.e. the average multiplicity (see Section 3). However, it is in the correlations between particles that the detailed information on the dynamics underlying their production may be expected to manifest itself.

High-order correlations have indeed been observed in all types of particle collisions at high energies in the form of super-Poissonian multiplicity fluctuations in small regions of phase space [31. With improving resolution, these fluctuations exhibit an approximate power-law scaling analogous to that observed for fractal objects, where the power is directly related to the anomalous dimension. The order dependence of this anomalous dimensions leads to the conclusion that particle production in a QCD jet follows a self-similar multifractal pattern, as in fact expected for a multiplicative branching process like QCD showering [32]. Deviations from exact scaling exist, however, in the form of saturation at small virtuality, due to the running of the coupling constant.

Detailed QCD predictions exist on the parton level for the so-called $H_{q}$ moments [33] measuring the relative amount of genuine $q$-particle correlation, on the one hand, and for the scaling behavior in angular [34] and (transverse) momentum variables [35], on the other. Assuming LPHD to also hold for these correlations, the calculations have been tested on the particle level in the L3 [26, 36], DELPHI [27] and ZEUS [28] experiments. While the JETSET Monte Carlo model perfectly reproduces the features of the data, the analytical calculations have to go as far as NNLA to qualitatively obtain the oscillation pattern of the $H_{q}$ moments observed for increasing rank $q$. This and the failure to reproduce the scaling behavior in angular variables may

\footnotetext{
${ }^{\ddagger}$ Author: W. Kittel
} 
be explained by the various approximations required in the analytical calculations, in particular the lack of energy-momentum conservation at each branching. However, the limit of validity of LPHD is reached when trying to reproduce the fluctuation pattern as a function of the limit on the (transverse) momentum. Since the analytical calculations are for partons at asymptotic energies, the predictions will be more readily tested at LHC energies.

\section{Jet Shape Observables}

Over the last few years the theory and phenomenology of jet shape observables has attracted a lot of attention. Nowadays, when jet shapes in $e^{+} e^{-}$annihilation have been analysed theoretically, both perturbatively [37] and at the level of the leading non-perturbative $1 / Q$ power corrections [38], the focus has shifted to the more complicated environment of DIS [39, 40, 41] and hadron-hadron collisions [42].

In particular, new results have been obtained by the Milan group for the out-of-plane QCD radiation in three-jet events in DIS [41] and in hadronic collisions [42], and were presented at the Workshop by Banfi and Smye respectively. The perturbative analyses have been carried out for the first time in hadronic collisions involving more than two emitting jets, at the next-toleading (NLL; single-logarithmic, SL) accuracy. The novel feature of the power-suppressed nonperturbative corrections to observables, involving hadrons in the initial state, is their dependence on the initial parton distributions, which enters at the SL level. As discussed by Dasgupta, this same feature has been found for the first time in the study of jet broadening in DIS [40], whose properties were already known to be subtle in the simpler $e^{+} e^{-}$annihilation case [43].

Recently the question about the actual need for non-perturbative $1 / Q$ power corrections has been raised by Hamacher. He has demonstrated that, at least according to the DELPHI analysis [44], a broad set of mean values of jet characteristics can be consistently described within the so-called renormalisation-group improved (RGI) scheme for dealing with the perturbative series, without invoking any explicit $1 / Q$ terms. However the RGI-perturbative approach itself has limited power, as it applies only to the mean values and only to $e^{+} e^{-}$annihilation, i.e. to one-parameter problems. In particular, it does not apply either to event shape distributions or the mean values in DIS and/or hadron-hadron collisions. Nevertheless, the amazing accuracy of the RGI-perturbative treatment of the $e^{+} e^{-}$mean shape observables poses a challenge for theorists: why does this specific prescription of guessing the higher-order perturbative terms seem to be so much more reliable than the other schemes.

The determination of resummed distributions of event shape variables and jet-rates has up to now proved very labour-intensive. This is despite the fact that many aspects of the calculations are actually fairly similar from one observable to another - for example one can usually make the same approximation concerning the multi-particle matrix elements and also exploit the same factorisation techniques. Zanderighi described an attempt at a numerical, completely general approach [45]. The main idea of this approach is to relegate a hard but routine theoretical analysis (all order resummation of double and single logarithms, matching with the exact matrix element calculation, etc.) of, virtually, all arbitrary collinear- and infrared-safe jet observables, to numerical calculation on a computer.

\footnotetext{
${ }^{\S}$ Authors: Yu.L. Dokshitzer and G. Zanderighi
} 


\section{BFKL Physicst}

One of the most interesting and challenging frontiers of perturbative QCD is provided by hadronic scattering processes in the so-called high-energy limit, i.e. asymptotically large centreof-mass scattering energy but fixed and finite momentum transfer. In QCD, the description of this regime is provided by the BFKL approach [46]. Although a relatively new field, there has been remarkable progress in both theoretical understanding and calculations and in experimental tests.

\subsection{BFKL at NLO: status and outlook}

Fadin [47] gave an overview of the BFKL approach to QCD processes in the high-energy limit, highlighting the achievements to date and some outstanding issues. The approach is based on one of the remarkable properties of QCD - gluon Reggeisation - which is extremely important for high energy QCD. The Pomeron, which determines the high energy behaviour of the cross sections, and the Odderon, responsible for the difference of particle and antiparticle cross sections, appear in this approach as compound states of two and three Reggeised gluons respectively. It is valid not only in the leading logarithmic approximation (LLA), where the BFKL approach was first developed, but also in the next-to-leading approximation (NLA). The scattering amplitude for the high energy process $A+B \rightarrow A^{\prime}+B^{\prime}$ at fixed momentum transfer $\sqrt{-t}$ in the NLA, as well as in LLA, is given by the convolution of impact factors $\Phi_{A^{\prime} A}$ and $\Phi_{B^{\prime} B}$, that describe the transitions $A \rightarrow A^{\prime}$ and $B \rightarrow B^{\prime}$ and the Green's function for the two interacting Reggeised gluons. Both the kernel of the integral equation satisfied by the Green's function and the impact factors are unambiguously defined in terms of the gluon Regge trajectory and the effective vertices for the Reggeon-particle interactions, which have been calculated at next-to-leading order (NLO) in a series of papers. The explicit form of the NLO kernel for forward scattering (with colourless exchange in the $t$ channel) was found. The cancellation of the infrared singularities in the kernel and in the impact factors of physical (colourless) particles was demonstrated. At the parton level (for quarks and gluons) impact factors were calculated in the NLO approximation for arbitrary momentum transfer $t$ and any colour state in the $t$-channel. For the non-forward kernel the only piece which is not yet calculated is the contribution of the two-gluon production for the colour singlet in the $t$-channel. The colour octet kernel is found for arbitrary $t$. The compatibility of the gluon Reggeisation with the $s$-channel unitarity ("bootstrap" of the gluon Reggeisation) was proved.

Two important problems remain unsolved. One is the calculation of the only missing piece in the non-forward kernel - the contribution of two-gluon production; the second is the calculation of the impact factors for highly virtual photons. The knowledge of the non-forward kernel means a significant extension of the region of possible phenomenological applications. The virtual photon impact factors play a specific role since they can be calculated "from first principles" and since there are experimental data for the high energy $\gamma^{*} \gamma^{*} \rightarrow$ hadrons cross section.

The calculation of the NLO BFKL kernel revealed a new problem - the large size of the scale-invariant correction to the eigenvalue function of the kernel, and also initiated a new wave of papers devoted to an old one - the running of the coupling. Several ways of solving the first problem are suggested. Possible ways of including the running coupling and its implications have also been widely discussed.

\footnotetext{
"Authors: J.R. Andersen and W.J. Stirling
} 


\subsection{Monte Carlo approach to studying the BFKL chain}

Andersen presented results on forward jet and $W$ production at hadron colliders obtained using a BFKL Monte Carlo model first reported in Ref. [48]. The basic idea of the Monte Carlo BFKL model is to solve the BFKL equation while maintaining kinematic information on each radiated gluon. This is done by unfolding the integration over the rapidity-ordered BFKL gluon phase space and introducing a resolution scale $\mu$ to discriminate between resolved and unresolved radiation. The latter combines with virtual corrections to form an IR safe integral. Thus the solution to the BFKL equation is recast in terms of phase space integrals for resolved gluon emissions, with form factors representing the net effect of unresolved and virtual emissions.

The benefit of using the BFKL MC is that it takes account of both energy and momentum conversation in hadronic 'BFKL' processes and also the inclusion of effects from the running of the coupling. In contrast, the analytic solution of the BFKL equation requires the integration over the full rapidity ordered phase space to obtain the parton level cross section. When it comes to including the parton fluxes from protons through the parton distribution functions, the contribution from the BFKL gluons to the Bjorken $x$ 's is therefore inaccessible in the standard approach. This means that in practice only the contribution to the subprocess centre-of-mass energy from the leading jets in (Mueller-Navelet) dijet production with a BFKL gluon ladder exchange can be taken into account in the standard BFKL approach. This inevitably leads to an overestimate of the parton flux. The BFKL MC approach also enables the details of the final state to be investigated.

The main result of the study [49] is that the contribution of the BFKL gluon radiation to the parton momentum fractions (at LHC energies) lowers the parton flux in such a way as to approximately cancel the rise in the subprocess cross section with increasing dijet rapidity separation $\left(\hat{\sigma}_{j j} \sim \exp (\lambda \Delta y)\right)$ predicted from the standard BFKL approach. The leading-order QCD prediction for the dijet cross section is therefore only slightly modified to an almost nochange situation compared to leading order QCD. However, other BFKL signatures such as the dijet azimuthal angle decorrelation do still survive.

Although at hadron colliders the simplest process exhibiting BFKL behaviour is the production of dijets with large rapidity separation, the formalism also applies to the production of more complicated forward final states. In particular, Andersen discussed the situation where one of the forward Mueller-Navelet jets is replaced by a $W$-jet pair, which also provides a testing ground for BFKL signatures [50]. In fact, the suppressing effect of the BFKL gluon radiation on the pdfs is less pronounced in this case, since requiring a $W$ in the final state means that at least one of the initial state partons must be a quark, with a less steeply falling pdf. This means that the BFKL rise in the partonic cross section is not compensated to the same extent as in the dijet case when including the pdfs to calculate the hadronic cross section.

\subsection{The Linked Dipole Chain model}

Gustafson reported on work with Miu [51] on the approach to BFKL asympototic behaviour in the framework of the Linked Dipole Chain Model (LDCM) [52]. The starting point is the observation that the small- $x$ structure function $F_{2}$ measured at HERA is well parameterised by the form $F_{2} \sim c\left(Q^{2}\right) x^{-\lambda\left(Q^{2}\right)}$, which resembles the asymptotic BFKL form apart from the fact that the effective exponent is $Q^{2}$ dependent: $\lambda\left(Q^{2}\right) \propto \ln \left(Q^{2} / \Lambda^{2}\right)$. The region of validity of this simple parameterisation is $10^{-4}<x<10^{-2}$. In the $x \rightarrow 0$ limit, the LDCM exhibits

the standard factorising BFKL behaviour, $F_{2} \sim x^{-\lambda_{\mathrm{BFKL}}}$, and interpolates smoothly into the 
standard DGLAP behaviour at higher $x$. In particular, the model agrees well with the $F_{2}$ data. The question then is what features of the LDCM are essential for the observed simple behaviour of the data.

The behaviour is understood in terms of a simple interpolating model for small $x$ based on the LDCM that has a smooth transition between large $k_{T}$, where ordered chains dominate, and small $k_{T}$ where non-ordered chains are most important. In the LDCM, the possibility to "go down" in transverse momentum along the chain, from $\kappa=\ln \left(k_{T}^{2}\right)$ to a smaller value $\kappa^{\prime}=\ln \left(k_{T}^{\prime 2}\right)$ is suppressed by a factor $\exp \left(\kappa-\kappa^{\prime}\right)$. The effective allowed distance $\delta$ for downward $\ln \left(k_{T}\right)$ steps is therefore at most about one unit, and the phase space factor $\kappa^{N} / N$ is replaced by $(\kappa+N \delta)^{N} / N$. The expression for the structure function $\mathcal{F}$ is obtained (for fixed coupling) by combining this with additional factors of $[\bar{\alpha} \ln (1 / x)]^{N} / N$, and summing over $N$. Simple algebra then shows that the DGLAP and BFKL behaviours are produced in the large and small $\kappa$ regions respectively. Further details and numerical studies can be found in Ref. [51].

\subsection{QCD and LHC: the experimental viewpoint}

Although the primary physics goal of the LHC is to search for the Higgs boson and signs of physics beyond the Standard Model, there will also be the opportunity to perform many highprecision, high-energy tests of QCD. A comprehensive overview of the machine, the detectors and the physics was given by Tapprogge.

The ATLAS and CMS general purpose detectors (GPDs) have been optimised for high $p_{T}$ signatures associated with discovery physics. However, they will at the same time, and without additional resources, allow precise QCD-related measurements. Indeed, a detailed understanding of QCD will be of the utmost importance for the discovery and measurements of any new physics. With an overall proton-proton collision energy of $14 \mathrm{TeV}$, the LHC will study the strong interaction in an as yet unexplored kinematic regime. Through processes involving the production of jets, prompt photons, electroweak gauge bosons and heavy quark flavours, the strong coupling will be measured up to TeV scales, partonic structure will be probed at very large $Q^{2}$ and small $x$, and all-orders resummation of large logarithms in 'two-scale' kinematic regimes will be tested.

However in order to exploit the new energy frontier to the full, some extensions to the detectors will be necessary. In particular, the GPDs have been designed and optimised for high $-p_{T}$ signatures in the central region $(|\eta|<5)$. There is considerable theoretical interest in "forward physics", much of which goes beyond the current reach of the GPDs in that it requires the detection of leading protons, particles and jets beyond $|\eta|=5$, and extended coverage of rapidity gaps. With such detector capability, one could measure

1. the total cross section

2. elastic scattering $\left(d \sigma / d t, \sigma_{e l} / \sigma_{t o t}, \rho\right.$ parameter $)$

3. diffractive scattering (single, double), e.g. $d^{2} \sigma / d t d M_{X}^{2}$

4. minimum bias event structure

5. properties of rapidity gaps (survival probability, as tools for new physics searches)

6. hard diffractive scattering (e.g. leading proton with high $p_{T}$ jets, $W$ bosons etc.) 
7. exclusive production of new heavy states, $p+p \rightarrow p+X+p$, where $M_{X}$ is measured precisely using the leading proton four momenta

8. two photon physics $p+p \rightarrow p+p+(\gamma \gamma \rightarrow X)$

The proposed TOTEM [53] experiment would provide appropriate forward physics capability, with roman pots for leading proton detection and forward telescopes covering $3<|\eta|<7$ for inelastic event measurements.

Another very interesting possibility is to identify and measure forward muons with, say, $|\eta|>5$. This would allow the Drell-Yan cross section $d^{2} \sigma^{\mu \mu} / d M^{2} d y$ to be measured at low dimuon mass $M$ and high rapidity $y$ [54]. Recalling that the parton momentum fractions are sampled (at leading order) at about $x_{1,2}=M / \sqrt{s} \exp ( \pm y$ ), this would enable very small $x$ parton structure to be probed. Designs for a forward " $\mu$ station" - a silicon-based detector inside the beam pipe - have been produced [55].

\section{References}

[1] N. Varelas, "QCD Results from D0", talk given at this workshop.

[2] A. Korytov, "Jet Fragmentation at CDF", talk given at this workshop.

[3] CDF Collab., T. Affolder et al., Phys. Rev. Lett. 87 (2001) 211804.

[4] Yu. Dokshitzer et al., Int. J. Mod. Phys. A7 (1992) 1875, Yu. Dokshitzer et al., Z. Phys. C55 (1992) 107.

[5] Ya. Azimov et al., Z. Phys. C27 (1985) 65, Ya. Azimov et al., Z. Phys. C31 (1986) 213.

[6] G. Corcella et al., JHEP 01 (2001) 010.

[7] D0 Collab., V. M. Abazov et al., Phys. Rev. D65 (2002) 052008.

[8] S. Catani et al., Nucl. Phys. B406 (1993) 187.

[9] R. Field, "The Underlying Event in Hard Scattering Processes", CDF/MINBIAS/PUBLIC/5746.

[10] I. Borozan, "An Eikonal Model for the Underlying Event in Hadronic Collisions", talk given at this workshop.

[11] T. Sjöstrand, "Production and Hadronization of Heavy Quarks", talk given at this workshop, E. Norrbin and T. Sjöstrand, Eur. Phys. J. C17 (2000) 137.

[12] R. Field, "The Sources of $b$-quarks at the Tevatron and their Correlations", UFIFT-HEP01-25 [hep-ph/020111]].

[13] WA82 Collab., M. Adamovich et al., Phys. Lett. B305 (1993) 402, E769 Collab., G. A. Alves et al., Phys. Rev. Lett. 72 (1994) 812, E791 Collab., E. M. Aitala et al., Phys. Lett. B371 (1996) 157.

[14] E. Norrbin and T. Sjöstrand, Phys. Lett. B442 (1998) 407. 
[15] J. Knapp, "Hadronic Particle Production in $10^{8} \mathrm{TeV}$ Cosmic Air Showers", talk given at this workshop.

[16] L. Lönnblad, "Correcting the Colour-Dipole Cascade Model with Fixed Order Matrix Elements," hep-ph/0112284.

[17] S. Burby, this workshop.

[18] A.P. Chapovsky, V.A. Khoze, A. Signer and W.J. Stirling, hep-ph/0108190.

[19] O. Biebel, Phys. Rep. 340 (2001) 165.

[20] V.A. Khoze, W. Ochs, J. Wosiek, in "At the frontier of particle physics - Handbook of QCD", ed. M. Shifman (World Scientific, Singapore 2001) p.1101.

[21] I.M. Dremin, "Some problems of the pQCD jet calculus", hep-ph/0201187.

[22] I.M. Dremin and J.W. Gary, Phys. Rep. 349 (2001) 301.

[23] M. Siebel, this workshop.

[24] N.H. Brook and I.O. Skillicorn, Phys. Lett. B479 (2000) 173, B497 55 (2001).

[25] ZEUS Collaboration, J. Breitweg et al., Eur. Phys. J. C12 (2000) 53.

[26] L3 Collaboration, M. Acciarri et al., Phys. Lett. B428 (1998) 186.

[27] DELPHI Collaboration, P. Abreu et al., Phys. Lett. B457 (1999) 368.

[28] ZEUS Collaboration, S. Chekanov et al., Phys. Lett. B510 (2001) 36.

[29] W.J. Metzger, this workshop.

[30] V. Kuvshinov, this workshop.

[31] A. Białas and R. Peschanski, Nucl. Phys. B273 (1986) 703; ibid. B308 (1988) 857;

E.A.De Wolf, I.M. Dremin and W. Kittel, Phys. Rep. 270 (1996) 1.

[32] G. Veneziano, Proc. $3^{\text {rd }}$ Workshop on Current Problems in High Energy Particle Theory, Florence 1979, eds. R. Casalbuoni et al. (Johns Hopkins University Press, Baltimore, 1979) p.45.

[33] I.M. Dremin, Physics-Uspekhi 37 (1994) 715.

[34] W. Ochs and J. Wosiek, Phys. Lett. B289 (1992) 159; ibid. B304 (1993) 144;

Yu.L. Dokshitzer and I.M. Dremin, Nucl. Phys. B402 (1993) 139;

Ph. Brax, J.-L. Meunier and R. Peschanksi, Zeit. Phys. C62 (1994) 649.

[35] S. Lupia, W. Ochs and J. Wosiek, Nucl. Phys. B540 (1999) 405.

[36] P. Achard et al. (L3 Collaboration ), "Measurement of the charged-particle multiplicity and inclusive momentum distributions in Z decays at LEP", submitted to Phys. Lett. B. 
[37] S. Catani, L. Trentadue, G. Turnock and B.R. Webber, Nucl. Phys. B407 (1993) 3;

S. Catani, G. Turnock and B.R. Webber, Phys. Lett. B295 (1992) 269;

S. Catani and B.R. Webber, Phys. Lett. B427 (1998) 377. Yu.L. Dokshitzer, A. Lucenti, G. Marchesini and G.P. Salam, JHEP 01 (1998) 011.

[38] M. Beneke and V.M. Braun, Nucl. Phys. B454 (1995) 253;

R. Akhoury and V.I. Zakharov, Phys. Lett. B357 (1995) 646; Nucl. Phys. B465 (1996) 295

G.P. Korchemsky and G. Sterman, Nucl. Phys. B437 (1995) 415;

P. Nason and M.H. Seymour, Nucl. Phys. B454 (1995) 291;

Yu.L. Dokshitzer, G. Marchesini and B.R. Webber, Nucl. Phys. B469 (1996) 93;

Yu.L. Dokshitzer, A. Lucenti, G. Marchesini and G.P. Salam, Nucl. Phys. B511 (1998) 396, erratum ibid. B593 (2001) 729; JHEP 05 (1998) 003.

[39] V. Antonelli, M. Dasgupta and G.P. Salam, JHEP 02 (2000) 001.

[40] M. Dasgupta and G.P. Salam, hep-ph/0110213.

[41] A. Banfi, G. Marchesini, G. Smye and G. Zanderighi, JHEP 11 (2001) 066;A. Banfi, G. Marchesini and G. Smye, in preparation.

[42] A. Banfi, G. Marchesini, G. Smye and G. Zanderighi, JHEP 08 (2001) 047.

[43] Yu.L. Dokshitzer, G. Marchesini and G.P. Salam, Eur. Phys. J. C3 (1999) 1.

[44] DELPHI Collaboration, Ralf Reinhardt et al., " A study of the energy evolution of event shape distributions and their means with the DELPHI detector at LEP", to appear in Eur. Phys. J. C;

P. Abreu, et al. (DELPHI Collaboration) Eur. Phys. J. C14 (2000) 557;

P. Abreu et al. (DELPHI Collaboration), Phys. Lett. B456 (1999) 322;

DELPHI Collaboration, DELPHI 2000-116 CONF 415, July 2000.

[45] A. Banfi, G.P. Salam and G. Zanderighi, JHEP 01 (2001) 018.

[46] V.S. Fadin, E.A. Kuraev and L.N. Lipatov, Phys. Lett. B60 (1975) 50;

E.A. Kuraev, L.N. Lipatov and V.S. Fadin, Sov. Phys. JETP44 (1976) 443;

Ya.Ya. Balitskii and L.N. Lipatov, Sov. J. Nucl. Phys.28 (1978) 822.

[47] A more complete discussion and a full set of references can be found in: V. S. Fadin, Nucl. Phys. A666 (2000) 155.

[48] L.H. Orr and W.J. Stirling, Phys. Rev. D56 (1997) 5875.

[49] J.R. Andersen, V. Del Duca, S. Frixione, C.R. Schmidt and W.J. Stirling, JHEP 0102 (2001) 007.

[50] J.R. Andersen, V. Del Duca, F. Maltoni and W.J. Stirling, JHEP 0105 (2001) 048.

[51] G. Gustafson and G. Miu, Eur. Phys. J. C23 (2002) 267. 
[52] B. Andersson, G. Gustafson and J. Samuelsson, Nucl. Phys. B467 (1996) 443;

H. Kharraziha and L. Lonnblad, JHEP 9803 (1998) 006;

B. Andersson, G. Gustafson, H. Kharraziha and J. Samuelsson, Zeit. Phys. C71 (1996) 613 ;

B. Andersson, G. Gustafson and H. Kharraziha, Phys. Rev. D57 (1998) 5543.

[53] For more information see the TOTEM web site at http://totem.web.cern.ch/Totem/

[54] See for example A. De Roeck, "Parton Density Measurements at Low $x$ at the LHC", Proc. Workshop on Forward Physics and Luminosity Determination at LHC, Helsinki, Finland, eds. K. Huitu et al., World Scientific (2001), p. 86.

[55] See for example V.P. Nomokonov, "The Microstation Concept for Forward Physics", Proc. Workshop on Forward Physics and Luminosity Determination at LHC, Helsinki, Finland, eds. K. Huitu et al., World Scientific (2001), p. 164. 